\title{
Pathogenetically based integrative therapeutic strategies in management of autism spectrum disorders
}

\author{
Dmitry Klokol ${ }^{1,2 *}$, Mike K.S. Chan ${ }^{1,2,3}$, Michelle B.F. Wong ${ }^{2,3}$, Dina Tullina ${ }^{2}$, Vladimir Chernykh ${ }^{1,2}$, Yee Kok Wah ${ }^{1,4}$ and Jere Rivera-Dugenio $^{5}$ \\ ${ }^{1}$ European Wellness Centers International, EU, APAC \\ ${ }^{2}$ Stellar Biomolecular Research, Germany \\ ${ }^{3}$ Fetal Cell Technologies International, Germany \\ ${ }^{4}$ Center of Integrative Medicine, Malaysia \\ ${ }^{5}$ Center of Integrative Medicine, Nevada, USA
}

\begin{abstract}
Autism and Autism spectrum disorders are the neurodevelopmental conditions characterized by persistent deficits in social communication and restricted repetitive behaviors. In spite of its occurrence being increasing from year to year, until now there is no unified opinion about etiology and pathogenesis of ASD. There is no widely accepted medical treatment for ASD as well. Authors summarized relevant data on the etiology of ASD, radiological findings in brain that persistent in autistic individuals, and proposed a pathogenetically based treatment protocol that implements a holistic integrative approach. The article summarizes therapeutic protocols proposed for complementary therapy of ASD that includes biomolecular therapies, immunotherapy, physiotherapy, speech therapy, nutrition and psychological support.
\end{abstract}

\section{Introduction}

Autism and the entire group of Autism spectrum disorders (ASD) are the neurodevelopmental conditions characterized by persistent deficits in social communication and restricted repetitive behaviors. American Psychiatric Association has recently made certain changes into the classification and diagnostic criteria of ASD, by removing some of the previously existing syndromes or combining them into one dimensional diagnosis [1].

The word Autismus derives from the Greek word autós (aủtó,, meaning "self"), and was first introduced by Swiss psychiatrist Eugen Bleuler in 1911 as an attempt to give a new description to schizophrenia - "autistic withdrawal of the patient to his fantasies, against which any influence from outside becomes an intolerable disturbance". One of the early diagnostic criteria for autism was formulated by Kanner (1943). It included (1) a profound lack of affective contact with people, (2) an anxiously obsessive desire for the preservation of sameness in routine and environment, (3) a fascination for objects that are handled with skills in fine motor movement, (4) repetitive and ritualistic behavior, and (5) difficulty with interpersonal verbal and non-verbal communications. A year later a similar, but milder condition was described by Hans Asperger. The latest change in the classification implemented by American Psychiatric Association has derecognized Asperger's syndrome as a separate nosological entity and integrated it into ASD group [1-3].

Lately the three criteria of ASD: (1) qualitative impairment in social interaction (2) in communication and (3) restricted repetitive and stereotyped patterns of behavior, interests, and activities have been reconstructed into two: (1) persistent deficits in social communication and social interaction (2) restricted, repetitive patterns of behavior, interests, or activities [1,2].
Outlook of the etiology and pathogenesis of ASD. Role of genetics and immunity in development of autism and ASD

For the past decade a tremendous increase in occurrence of ASD was noticed worldwide, with the two leading counties being USA and China. According to the Centers for Disease Control and Prevention report, ASD affected 1 in 68 children in the United States in 2014; and 1 in 50 in 2016 (Chart 1). Albeit increased global prevalence of autism its etiology is not yet completely understood. Genetic theories and role of environmental factors in etiology of autism are being considered [4].

Until now there is no unified opinion about pathogenesis of autism as well $[3,5]$. It appears, most likely, that ASD is a multifactorial disorder occurring in complex ways. Perhaps, there are several subtypes of ASD each with differing etiologies. For instance, Lainhart et al. (2002) reported of a specific sub-type of ASD that occurs in 20-30\% of cases and is characterized by normal initial development followed by regression occurring after age of 1.5-2 years old. On the contrary, most cases of ASD have consistent delayed development [6].

Genetics are believed to play certain role in development of ASD, however does not explain recent drastic increase in reported cases. If one identical twin is diagnosed with ASD, then the other one has

Correspondence to: Dmitry Klokol, Stellar Biomolecular Research, An der Welle 4,60322 Frankfurt am Main, Germany, Tel: +60169687977; E-mail: dr.dmytro@ sbi-europe.com

Key words: autism, ASD, stem cells, biomolecular therapy, peptide therapy, immunotherapy, fetal cell transplantation, cell therapy

Received: May 14, 2017; Accepted: June 29, 2017; Published: July 03, 2017 


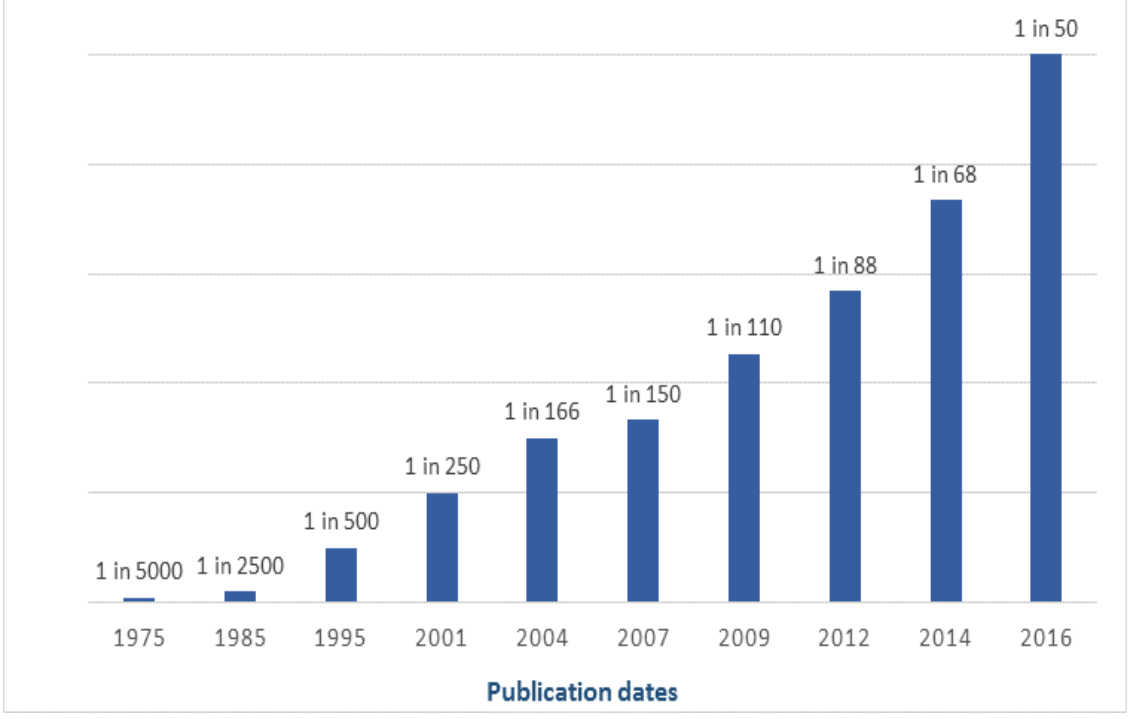

Chart 1. Statistics of changes in ASD occurrence over the past three decades (USA).

$30-40 \%$ chance to develop same disorder. According to other sources probability rates is $90 \%$ for identical twins and $10 \%$ for non-identical [7-9]. Few studies found ASD linked to maternal exposure to certain viruses (measles, mumps, rubella, herpes, syphilis, cytomegalovirus and toxoplasmosis) and chemicals (thalidomide and valproic acid) [10-12]. Some of the chemicals contained in soap and body washes, in particular methylisothiazolinone, affect neural development of the child both during intrauterine period as well as in early childhood. There are also evidences that some vaccine components may be linked to ASD $[11,12]$.

In recent years another theory proposing role of immune dysfunction, oxidative stress, and mitochondrial dysfunction in autism is gaining popularity [13-15]. This theory is supported by the data that immunosuppression and oxidative stress observed in ASD could be associated with active pathogens from the perinatal period and early terms of child development. Viral infections and dysbacteriosis are also known to disrupt mitochondrial activity in cells $[16,17]$.

\section{Role of imaging studies and biological markers in au- tism and ASD and its links to symptomatology}

The exact nature of the anatomical changes happening in the autistic brain is also not completely understood. Various studies have shown differences in development in many brain areas including frontal and temporal lobes, cerebellum, sub-cortical amygdala, and hippocampus. Other studies mention disruption in connectivity between and within brain areas rather than specific location [18-20].

Due to the lack of consent about etiology of autism and ASD, there is no widely accepted medical treatment for it yet.

Magnetic Resonance Image (MRI) study can provide direct information on neurodevelopmental characteristics underlying ASD. Structural MRI may reveal abnormalities in gray and white matter and some regional brain differences between ASD and non-ASD brain. The most constant finding is an increased total brain volume growth in children with autism between 2 to 4 years of age. Brain development during early childhood in ASD is predominantly due to enlarged brain volume of the frontal and temporal lobes, combined with delayed growth and declined volumetric capacity of the brain after 10-15 years of age. Accelerated expansion of cortical surface area of the gray matter in ASD appears to be associated with impaired maturation of the white matter (Figure 1).

Pertaining to the brain enlargement, its pathological mechanism is still unknown. Specific brain areas are correlating with clinical phenotypes of autism - frontal, temporal, parietal lobes, amygdala, hippocampus, basal ganglia, and anterior cingulate cortex. Abnormalities detected in Wernicke's and Broca's area, frontal lobe, amygdala and superior temporal sulcus are related to defects in language development and social behavior (Figure 2). Functional MRI is able to reveal brain areas with atypical brain functions $[18,19]$.

Numerous brain imaging studies have examined other aspects of the cerebral cortex, such as cortical shape and sulcal patterns. Atypical cortical folding in the brains of children with ASD was observed. There is significantly enlarged gyrification of the frontal lobe in individuals with ASD. Regional cortical folding is increased in bilateral posterior brain regions in juveniles and adults with ASD. Whereas, reduced local gyrification has been reported in the right inferior frontal and medial parieto-occipital cortices in children with ASD and in the left supramarginal gyrus in adult individuals with ASD $[18,19]$.

It is interesting how cortical thickness changes with age. Zielinski et al. measured cortical thickness in various regions of ASD brains and reported progressive cortical thinning in individuals with ASD after the age of 3 until 40 years. Individuals with ASD usually experience thinning of cortices and reduction of surface area with age. In other words, abnormal cortical expansion during early childhood is followed by rapid cortical thinning during adolescence and adulthood [20].

The symptomatology of ASD is mostly related to difficulties with social communication and interaction. The main symptoms are associated with social issues (no interest in other children playing, vicious with siblings); communication issues (avoids eye-contact, unaware of environment); bizarre and repetitive behaviors (staring at ceiling fan, flapping, lining up toy cars). ASD symptoms also include motor issues - fine motor deficits, poor coordination, toe-walking; sensory issues - impossibility to self-bathe, difficulty tolerating music; 


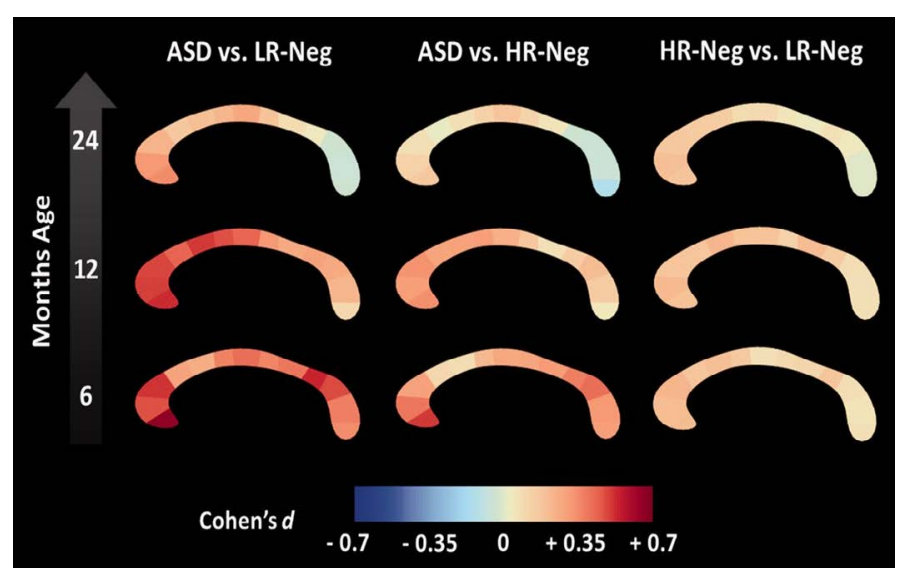

Figure 1. In a prospective, longitudinal study of infants with elevated risk of autism spectrum disorder, Wolff et al. reveal increased corpus callosum area and thickness in those who later develop the disorder. Diffusion tensor imaging data suggest that these anatomical differences may result from abnormalities in neurodevelopmental processes specific to infancy (from Wolff JJ. Altered corpus callosum morphology associated with autism over the first 2 years of life. Brain (2015) 138 (7): 2046-2058).

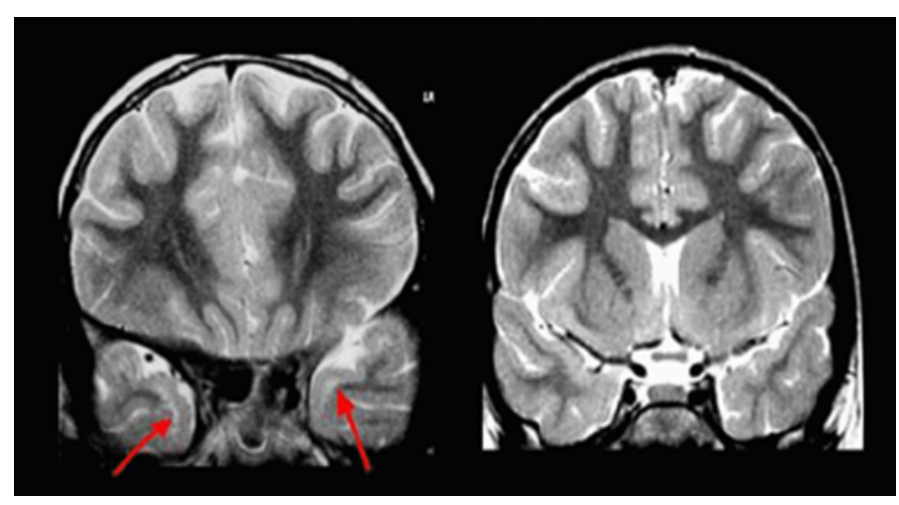

Figure 2. Temporal pole abnormality characteristic for ASD. Autistic child (left) versus non-autistic child's brain (right).

self-injurious behaviors - head-banging, self-biting, no sense of danger, no fear of heights. Children with ASD may have difficulty processing auditory information. A child with autism may have extreme difficulty tolerating music, noise, textures and new experiences or environments. They may go days without any apparent need to sleep, may not seem to notice difference between day and night [1].

Many holistic doctors propose measurement of biomarkers related to immune dysregulation as a part of ASD evaluation in a clinical setting. Recently, evaluation of the activity of alpha- $\mathrm{N}$-acetylgalactosaminidase (Nagalase) has been made commercially available as a diagnostic laboratory measurement. It is a nonspecific biomarker, which appears to be an important indicator of secondary immunosuppression [21,22] (Figure 3).

\section{Integrative therapeutic protocol for autism and ASD. New prospective for treatment}

As we already mentioned, there is no commonly accepted treatment for autism. However, taking into account all the latest progress made in understanding the nature of ASD, we have discovered new, more effective ways of managing autism. Conventional approach stresses on education, behavioral strategies and facilitative therapies that target communication, social skills, promotes academic potential, and manages maladaptive behavior [3,5]. Generally accepted medications are not been able to correct core deficits of autism.
Prof. Abdul Halim has written in his recent book "Hope for untreatable medical disorders. Live cell therapy explained" that "treatment of the autistic child will take a lot of effort, time, and financial resources. The applied behavior analysis program is often offered for autistics. The program trains child to develop verbal communications, develop and imitate social skills, and reduce stereotypic behavior. The triggers for negative behavior and why it persists are noted, and through appropriate reward, the behavior is not reinforced or modified. This program can be very intensive, and speech therapy is often incorporated. These alone will not be adequate without looking into the biological parameters and needs of the child" [23].

So what will be adequately addressing biological parameters and needs of the child with ASD?

Since last decade live precursor cell therapy has been applied as a treatment for more than 800 cases of ASD with remarkable results. From our latest clinical experience we have learnt that precursor stem cell (PSC) therapy is capable of brining improvements not only in early childhood, as it was previously thought, but also in adults with autism, who were not treated timely in the past [24,25].

During neurogenesis neural progenitor cells express regulatory genes that subdivide each area of the brain into compartments and regulate their size. Progenitor cells generate neurons during early to mid-embryogenesis. Neurons progressively migrate to their target sites, some of them carrying with them the legacy of transcription factors expressed by their progenitors [26,27].

Precursor stem cells are specialized organ-specific stem cells that structurally and functionally belong to exact area of the central nervous system. The real challenge for the physician is to understand core aspects of physical, physiological, social and psychological disturbances of the individual with ASD and be able to pick up peculiarities of each and every case. Success of the treatment depends on the correct prescription of necessary types of cells. The entire treatment is a dynamic process that often requires reformulation of the protocol and repeated applications of live precursor cells. Usually typical prescription includes corpus

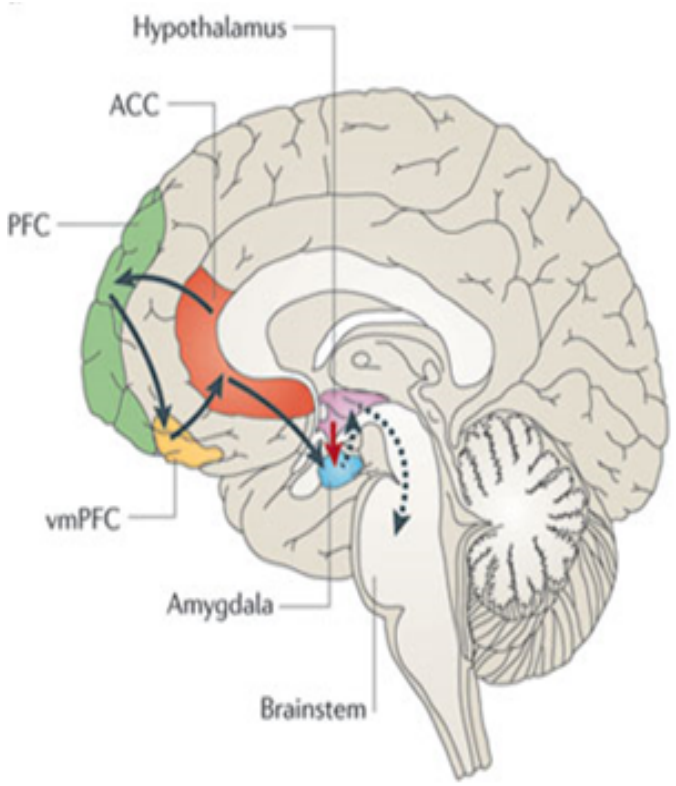

Figure 3. Relevant areas of brain that should be addresses with biomolecular therapies in a complex management of ASD. 
collosum, amygdala, hippocampus, medulla alba, temporal and frontal lobes as a basic protocol with different variations, depending on the peculiarities of each case $[23,24]$.

Patients after therapy with PSC in short time learnt to independently regulate their own behaviors and act appropriately at home, school, family, and community based situations. In most of the cases, within first two weeks patients show positive changes in their social and language development. After 2-3 months children begin to eye-to-eye contact, increase their attention span, respond to verbal instructions and develop meaningful speech. Children become more active, show more focus and interest in the environment. After 6 months they become more fluent in communicating with others and independent with the self-management [23].

After repeated treatment patients are able to manage anxiety, mood problems, attention deficits, depression, and further develop their social skill. At the same time PSC therapy manages patient's problems with seizures, gastrointestinal disorders, dietary imbalances and disrupted sleep patterns.

To maximize the efficacy of PSC therapy we introduced neuropeptides - cellular extracts from the central nervous system as a second stage of the treatment protocol for the ASD. This neuropeptides play important role in modulating the higher nervous activity, including conditioned and unconditioned reflexes, higher mental functions that ensure adequate behavior in changing surrounding natural and social conditions. CNS peptides act as essential mediators of emotional and social behavior, speech and intellectual development.

Years of research and extensive global practice has proven high effectiveness of Nano Organ Specific CNS peptides by MF+ (SBI, Germany). Formulation and manufacturing technology of the Nano organ specific peptides were first discovered and invented by MF3/ MF+ Research \& Development unit in 2004. More than a decade of world-wide application of MF3/MF+ Nano Cell Extracts has proven its absolute safety, high bioavailability and outstanding efficacy.

In 2015 SBI Research \& Development unit (Germany) has completed formulation of the principally new range of cell extracted peptides, which is produced under $\mathrm{MF}^{\mathrm{Tn}}$ brand and known as "Mito Organelles ${ }^{\mathrm{Tm} \omega}$. Mito Organelles ${ }^{\mathrm{Tm}}$ of MF+ supersedes all other commercially available organ-specific peptides on the World's market in terms of purity of content, concentration of peptides, and, inevitably, clinical efficacy.

The administration of CNS-specific cell extracted peptides Mito Organelles $^{\mathrm{Tm}}$ of MF+ (SBI, Germany) as the maintenance phase of treatment following the PSC therapy has demonstrated dramatic reduction of anxiety and significant improvement in behavioral aspects in patients with autism. Mito Organelles ${ }^{\mathrm{Tm}} \mathrm{CNS}$ peptides promote the confidence increase and reduction of aggressive behavior in autistic patients, improves their communication abilities and social adaptation skills. Adding pineal gland cell extracts (Mito Organelles ${ }^{\mathrm{Tx}}$ Pineal gland specific peptides) to the treatment protocol helps to ameliorate sleep disorders in ASD patients.

Important to mention that after 4-6 months of therapy patients feel easier to sustain conversation, and demonstrate evidently less repetitive motor mannerisms and flexible adherence to routines. The positive effect on improvement of higher mental functions also included the development of peer relationships, clear presence of social or emotional reciprocity, progress on speech development and verbal communication.
The third component of our proposed treatment protocol for ASD is GcMAF Forte (Lab RMS). GcMAF Forte is the only in the World GcMAF-containing product formulated in combination with high-molecular weight hyaluronic acid that enables a slowrelease of macrophage activating factor and its constantly high serum concentration during one week after administration. GcMAF Forte (Lab RMS) is an immune enhancer that has important influences on immune, neural and endocrine systems. Plasma levels of endogenous GcMAF are lower in individuals with autism and ASD $[22,28]$.

Besides other immune disorders, decreased levels of total plasma IgG and IgM are commonly observed in individuals with autism. Its reduced levels correlated with behavior in such manner that individuals with the higher behavioral disturbances have the lowest IgG and IgM levels [29].

The improvement in ASD symptoms after the concurrent application of precursor stem cells, CNS specific peptides and GcMAF Forte should be observed within first 2 months after therapy. The recommended duration of GcMAF Forte administration is 4 months. It is important to provide an adequate nutritional support, speech and occupational therapy, psychological aid to the individuals with ASD. The therapeutic modalities described in this article should be applied along with other holistic treatments, i.e. electro-magnetic therapy, detoxification, hyperbaric oxygenation, transcranial electrostimulation, cyclotronic ionic resonance, etc.

Lately we have been working on integrating quantum medicine technologies into our holistic protocols. One of it is RASHA Morphogenetic Harmonizer System, developed in USA (Nevada). It combines the brilliant technologies of Nikola Tesla, Antoine Priorie' and Dr. Royal Rife into one integrative quantum self-healthcare system. The RASHA is controlled by one of the most advanced scalar and Rife frequency generating software technology. When operated and activated by the software, selected frequencies (transverse waves) are transmitted through the dual scalar spiral coils that are then pulsed into the custom plasma gas tube. Plasma can transform transverse waves into longitudinal, scalar waves. Additionally, plasmas can also create phase conjugate waves or time-reversed waves [30].

The RASHA Morphogenetic Harmonizer System is a three-fold approach system. The RASHA simultaneously harmonizes the two systems in the body concerned with prevention and management of a malady (immune system) and regeneration of the damaged cells from that said malady (cellular regenerative system). However, its main purpose is to reverse the mutations in the introns (or junk DNA) via reprogramming the morphogenetic encryption lattice of the introns [30].

\section{Conclusion}

In our future publications, we will emphasize on presenting statistical reports of the case studies with complete reversal of ASD symptoms after our treatment protocol.

This holistic integrative protocol of management of ASD has been formulated and implemented world-wide by European Wellness Centers $^{\mathrm{sm}}$ International Group with support from FCTI and SBI research and development unit [31].

There is no panacea for all diseases; there is no unique solution to all problems. In European Wellness International Group we believe in integral holistic approach to the management of health problems. In order to provide a successful solution to health problems we have built our commendable therapeutic paradigms as a powerful tool that addresses patient as a whole, as a self-regulating biological system. 


\section{Acknowledgments}

Authors would like to acknowledge contribution made to the research by our dedicated partners - FCTI (www.fetal-cells. com), world's largest provider of precursor stem cells, SBI (Stellar Biomolecular Innovations) research and development unit (www.sbieurope.com), and Lab RMS (www.labrms.com).

\section{References}

1. American Psychiatric Association (2013) Diagnostic and statistical manual of mental disorders. Washington DC, USA.

2. Myers SM, Johnson CP (2007) American Academy of Pediatrics, Council on Children with Disabilities. Management of children with autism spectrum disorders. Pediatrics 120: $1162-1182$

3. National Professional Development Center on Autism Spectrum Disorders (2011) Evidence-Based Practices.

4. Muhle R, Trentacoste SV, Rapin I (2004) The genetics of autism. Pediatrics 113: e472486. [Crossref]

5. Simpson RL (2005) Autism spectrum disorders: Interventions and treatments for children and youth. Thousand Oaks, CA: Corwin Press.

6. Lainhart JE, Ozonoff S, Coon H, Krasny L, Dinh E, et al. (2002) Autism, regression, and the broader autism phenotype. Am JMed Genet 113: 231-237. [Crossref]

7. Sarris M. Twins Study Finds Large Genetic Influence in Autism. Interactive Autism Network. Published on-line.

8. Hallmayer J, Cleveland S, Torres A, Phillips J, Cohen B, et al. (2011) Genetic heritability and shared environmental factors among twin pairs with autism. Arch Gen Psychiatry 68: 1095-1102. [Crossref]

9. Ronald A, Happé F, Bolton P, Butcher LM, Price TS, et al. (2006) Genetic Heterogeneity Between the Three Components of the Autism Spectrum: A Twin Study. J Am Acad Child Adolesc Psych 45: 691-699. [Crossref]

10. Libbey JE, Sweeten TL, McMahon WM, Fujinami RS (2005) Autistic disorder and viral infections. J Neurovirol 11: 1-10. [Crossref]

11. Gerber JS, Offit PA (2009) Vaccines and Autism: A Tale of Shifting Hypotheses. Clin Infect Dis 48: 456-461. [Crossref]

12. Madsen KM, Hviid A, Vestergaard M, Schendel D, Wohlfahrt J, et al. (2002) A Population-Based Study of Measles, Mumps, and Rubella Vaccination and Autism. $N$ Engl J Med 347: 1477-1482. [Crossref]

13. Goines P, Van de Water J (2010) The immune system's role in the biology of autism. Curr Opin Neurol 23: 111-117. [Crossref]

14. Ashwood P, Wills S, Van de Water J (2006) The immune response in autism: a new frontier for autism research. J Leukoc Biol 80: 1-15. [Crossref]
15. Enstrom AM, Van de Water JA, Ashwood P (2009) Autoimmunity in autism. Curr Opin Investig Drugs 10: 463-473.

16. Jyonouchi H, Sun S, Itokazu N (2002) Innate immunity associated with inflammatory responses and cytokine production against common dietary proteins in patients with autism spectrum disorder. Neuropsychobiology 46: 76-84. [Crossref]

17. Gupta S, Aggarwal S, Rashanravan B, Lee T (1998) Th1- and Th2-like cytokines in CD4+ and CD8+ T cells in autism. J Neuroimmunol 85: 106-109. [Crossref]

18. Courchesne E, Pierce K (2005) Why the frontal cortex in autism might be talking only to itself: local over-connectivity but long-distance disconnection. Cur Opinion Neurobiol 15: 225-230. [Crossref]

19. Catani M, Dell'Acqua F, Budisavljevic S, Howells H, Thiebaut de Schotten M, et al (2016) Frontal networks in adults with autism spectrum disorder. Brain 139: 616-630. [Crossref]

20. Zielinski BA, Prigge MB, Nielsen JA, Froehlich AL, Abildskov TJ, et al. (2014) Longitudinal changes in cortical thickness in autism and typical development. Brain 137: 1799-1812. [Crossref]

21. Cohly HH, Panja A (2005) Immunological findings in autism. Int Rev Neurobiol 71 317-341. [Crossref]

22. Bradstreet JJ, Vogelaar E, Thyer L (2012) Initial Observations of Elevated Alpha-NAcetylgalactosaminidase Activity Associated with Autism and Observed Reductions from GC Protein-Macrophage Activating Factor Injections. Autism Insights 2012 431-438.

23. Abdul Halim (2017) Hope for untreatable medical disorders. Live cell therapy explained. Leicester, UK, pp: 256.

24. Molnar ME (2006) Stem cell transplantation. A textbook of Stem cell xenotransplantation. FCTI. Medical and engineering publishers. USA, pp: 632.

25. Ichim TE, Solano F, Glenn E, Morales F, Smith L, et al. (2007) Stem cell therapy for autism. J Transl Med 5: 30. [Crossref]

26. Rubenstein JL, Puelles L (1994) Homeobox gene expression during development of the vertebrate brain. Curr Top Dev Biol 29: 1-63. [Crossref]

27. Shimamura K, Rubenstein JLR (1997) Regulation of patterning and differentiation in the embryonic vertebrate forebrain. In: Molecular and cellular approaches to neural development (Cowan WM, Jessell TM, Zipursky SL), pp. 356-390.

28. Yonk LJ, Warren RP, Burger RA, Cole P, Odell JD, et al. (1990) CD4+ helper T cell depression in autism. Immunol Lett 25: 341-345. [Crossref]

29. Heuer L, Ashwood P, Schauer J, Goines P, Krakowiak P, et al. (2008) Reduced levels of immunoglobulin in children with autism correlates with behavioral symptoms. Autism Res 1: 275-283. [Crossref]

30. Rivera-Dugenio J. Scalar Plasma Technology and Structured Marine Plasma Water as an Effective Reverse Aging Protocol.

31. Klokol D, Chan MKS, Wong MBF (2017) European wellness - the evidenced rationale behind the biological medicine: ad astra per aspera. J Pharm Biomed Sci 07: 19-22.

Copyright: $\odot 2017$ Klokol D. This is an open-access article distributed under the terms of the Creative Commons Attribution License, which permits unrestricted use, distribution, and reproduction in any medium, provided the original author and source are credited. 